\title{
EXIL ET DISCOURS SUR LA TRADUCTION
}

\author{
Winibert Segers \\ Katholieke Universiteit Leuven. Leuven, Bélgica/Université d'Angers, \\ Angers, França. \\ Henri Bloemen \\ Katholieke Universiteit Leuven. Faculty of Arts. Leuven, Flanders, \\ Belgium.
}

Résumé : Le Catéchisme préparatoire au baptême a été traduit dans différentes langues du Congo. Ces traductions ont été faites par des missionnaires catholiques flamands à la fin du dix-neuvième siècle et $\mathrm{au}$ début du vingtième siècle. Jules Garmyn a traduit Le Catéchisme préparatoire au baptême en tshiluba (1898) ; Camille Van Ronslé en bobangi (1898), en kikongo (1900) et en lingala (1911) ; Jules Van Houtte en lonkundu (1912). Les missionnaires étaient des traducteurs nonnatifs. Ils traduisaient vers des langues qui n'étaient pas leurs langues maternelles, vers des langues qu'ils avaient apprises en fonction de leur mission. Les missionnaires avaient bien compris que la traduction était un instrument puissant qui aidait à propager la foi. La traduction est faite dans une perspective fonctionnelle. Elle devait aider à changer radicalement les mœurs et coutumes des populations locales. Le Catéchisme préparatoire au baptême est un texte imprégné de la culture et de la tradition occidentales. Nous nous demanderons comment les missionnaires ont traité les spécificités lexicales et métaphoriques du texte source et comment les traductions ont pu fonctionner dans les cultures congolaises, cultures pour lesquelles le texte source est un élément profondément étrange. Ce thème ne sera abordé que succinctement. Il détournerait le lecteur du fond de notre contribution : la place de la notion d'exil dans le discours sur la traduction. La notion d'exil est souvent comprise de façon subjectivée. La notion est centrée sur l'auteur ou le traducteur qui se trouvent dans une situation d'exil. Antoine Berman et Walter Benjamin nous aiderons à essayer de comprendre la notion d'exil d'une autre façon, de façon 
désubjectivée. Les langues, les textes, l'original, la traduction se trouvent dans une situation d'exil. Nous nous demanderons ce que la notion d'exil pourrait apporter à la compréhension du mouvement - un mouvement de la Belgique vers le Congo - dans lequel le Catéchisme préparatoire au baptême est pris.

Mots-clés : Missionnaires. Langues du Congo. Désubjectivation. Antoine Berman. Walter Benjamin. Essence de la traduction.

\title{
EXILE AND DISCOURSE ON TRANSLATION
}

\begin{abstract}
The Catéchisme préparatoire au baptême has been translated into various languages of Congo. These translations were made by Flemish Catholic missionaries in the late nineteenth century and early twentieth century. Jules Garmyn translated the Catéchisme préparatoire au baptême in Tshiluba (1898); Camille Van Ronslé in Bobangi (1898), Kikongo (1900) and Lingala (1911); Jules Van Houtte in Lonkundu (1912). The missionaries were non-native translators. They translated into languages that were not their native languages, into languages they had learned according to their mission. The missionaries had understood that translation was a powerful instrument that helped to spread the faith. Translation is done from a functional perspective. It had to help to change radically the habits and customs of local people. The Catéchisme préparatoire au baptême is a text steeped in the Western culture and tradition. We will ask how the missionaries treated the lexical and metaphorical characteristics of the source text and how the translations functioned in Congolese cultures, cultures for which the source text is a deeply strange element. This theme will be discussed only briefly. It would divert the reader from the core of our contribution: the place of the notion of exile in discourse on translation. The notion of exile is often understood in a subjectified way. The notion is centered on the author or translator who are in a situation of exile. Antoine Berman and Walter Benjamin will help us to try to understand the notion of exile in another way, in a desubjectified way. The languages, the texts, the original, the translation are in a situation of exile. We will ask what the notion of exile could bring to the understanding of a movement - a movement from Belgium to Congo - in which the Catéchisme préparatoire au baptême is taken.
\end{abstract}

Keywords: Missionaries. Languages of Congo. Desubjectivation. Antoine Berman. Walter Benjamin. Essence of translation. 
Bina bepim' Eklesia ile nyama, bina benko ? Nde Vandredi inkuma, Samedi ie ntondo e Paska, la wuna ntondo y'Ebotwelo. (Catéchisme préparatoire au baptême traduit dans la langue des Nkundu [du Lac Léopold II] 32)

Ce passage est tiré du texte intitulé Catéchisme préparatoire au baptême traduit dans la langue des Nkundu (du Lac Léopold II). La traduction a été publiée à Bruxelles en 1912. Le titre de la traduction n'est pas un titre lonkundu. Le titre est un titre français, qui nous dit, en français, que le texte qui suit est une traduction. Dans le titre n'apparaît pas le nom de la langue cible, le lonkundu. Le titre renvoie à une des ethnies du Congo, les Nkundu, et à un endroit géographique, le Lac Léopold II. Le lac porte le nom du deuxième roi des Belges et est situé au centre-ouest du Congo.

Pour donner une idée du contenu du passage que nous venons de citer, nous le rétro-traduisons en français :

Quels sont les jours où l'Église interdit de manger de la viande?

Tous les vendredis, samedi avant Pâques, et le jour avant Noël.

La traduction dans la langue des Nkundu est un mélange de langues. La traduction lonkundu est une traduction métissée. Elle est truffée de mots français ('Vandredi', 'Samedi') et latins ('Eklesia', 'Paska'). L'orthographe des mots 'Vandredi', 'Eklesia' et 'Paska' a été adaptée à la langue cible. La traduction a sans doute eu un effet étrange sur les lecteurs habitant dans les environs du Lac Léopold II, l'actuel Lac Mai-Ndombe.

Nous utilisons le mot 'lecteurs', mais 'destinataires' serait plus approprié. Au début du vingtième siècle, les populations locales étaient en grande majorité analphabètes. Les premières écoles au Congo ont été fondées par des missionnaires.

L'effet étrange de la traduction lonkundu n'est pas seulement causé par la présence de mots français et latins, mais aussi par 
le contenu du texte, qui est très éloigné de la culture Nkundu. Le Catéchisme préparatoire au baptême nous parle entre autres du Pape, qui vit à Rome, d'Adam et Ève, que Dieu a chassés du Paradis terrestre et des anges, qui n'ont pas de corps et habitent au ciel. Le Catéchisme préparatoire au baptême devait être appris par cœur et voulait avoir un impact sur la vie des populations indigènes. Le passage que nous avons cité est un exemple de l'influence que le Catéchisme préparatoire au baptême voulait exercer sur les habitudes alimentaires.

Les mots français et latins sont des mots étranges dans le texte cible, des mots qui n'appartiennent pas au lexique lonkundu. Le traducteur a comblé les lacunes lexicales à l'aide de mots français et latins. Ces lacunes peuvent être individuelles, des mots lonkundu que le traducteur ne connaît pas, ou générales, des mots pour lesquels il n'y a pas d'équivalent dans la langue des Nkundu. Le comblement des lacunes lexicales est très visible à cause de la différence entre les mots lonkundu d'une part et les mots français et latins d'autre part.

Il est surprenant de constater que 'vendredis' et 'samedi' ont été repris presque littéralement dans la traduction. La langue des Nkundu, n'a-t-elle pas de mots pour désigner ces jours de la semaine ? Le champ sémantique du temps, est-il découpé de façon fondamentalement différente par rapport aux langues occidentales ? Les Nkundu, ont-ils une conception du temps qui diffère sensiblement de la conception occidentale ? N'employaientils pas le système de la semaine de sept jours ? Le système de la semaine de sept jours a-t-il été introduit par les missionnaires ? La réponse à ces questions de linguistique historique demanderait des recherches qui dépassent le thème de cette contribution.

Une autre hypothèse peut être émise pour expliquer le traitement de 'vendredis' et 'samedi'. Le traducteur a repris ces mots parce qu'il ne connaissait pas les mots correspondants lonkundu. Cette hypothèse nous paraît peu probable parce que le champ sémantique du temps est un des premiers champs que l'on découvre en apprenant une nouvelle langue. 
En ce qui concerne les fêtes chrétiennes, 'Pâques' et 'Noël', nous nous demandons pourquoi elles sont traitées différemment dans la traduction lonkundu. 'Pâques' devient 'Paska' ; 'Noël' devient 'Ebotwelo'. 'Paska' est un emprunt du latin 'pascua' ; 'Ebotwelo' est un mot lonkundu, qui signifie 'naissance'. Comment expliquer le traitement différent des mots 'Pâques' et 'Noël' ?

La traduction dans la langue des Nkundu est très accueillante à l'égard du français et du latin. 'Vandredi', 'Samedi', 'Eklesia' et 'Paska' ne sont pas les seuls mots français et latins dans la traduction lonkundu. Le traducteur utilise à plusieurs reprises les mots 'angelu', 'batisimu', 'eukaristia', 'grasia', 'katekisimu', 'penetensia', 'sakramentu' et d'autres mots français et latins qu'il adapte à la langue cible. L'emploi de mots français et latins crée une distance entre la traduction et les lecteurs Nkundu. En essayant de trouver des « équivalents » en lonkundu - 'équivalents', entre guillemets, évidemment -, le traducteur aurait facilité l'accès aux lecteurs. Peutêtre que le terme 'inculturation' pourrait s'appliquer à cette approche traductive. 'Inculturation' est un terme missiologique. L'inculturation est une adaptation de l'enseignement de la foi aux cultures locales. La culture locale, étrangère est intégrée dans la propre culture.

La traduction dans la langue des Nkundu, le lonkundu, n'est pas la seule traduction congolaise du Catéchisme préparatoire au baptême. Le Catéchisme préparatoire au baptême a été traduit dans différentes langues du Congo. Ces traductions ont été faites par des missionnaires catholiques flamands à la fin du dix-neuvième siècle et au début du vingtième siècle. Jules Garmyn ${ }^{1}$ a traduit Le Catéchisme préparatoire au baptême en tshiluba (1898) ; Camille Van Ronslé ${ }^{2}$ en bobangi (1898), en kikongo (1900) et en lingala (1911) ; Jules Van Houtte ${ }^{3}$ en lonkundu (1912). La traduction de Van Houtte est celle dont nous avons cité un passage ${ }^{4}$.

\footnotetext{
${ }^{1}$ Jules Garmyn (Beveren 1861 - Westvleteren 1926).

${ }^{2}$ Camille Van Ronslé (Lovendegem 1862 - Boma 1938).

${ }^{3}$ Jules Van Houtte (Kortrijk 1877 - Torhout 1966).

${ }^{4}$ Le nom du traducteur n'est pas mentionné dans le Catéchisme préparatoire au baptême traduit dans la langue des Nkundu (du Lac Léopold II). Mpia Bekina (38) attribue la traduction à Jules Van Houtte.
} 
Les missionnaires étaient des traducteurs non-natifs. Ils traduisaient vers des langues qui n'étaient pas leurs langues maternelles, vers des langues qu'ils avaient apprises en fonction de leur mission. Les missionnaires avaient bien compris que la traduction était un instrument puissant qui aidait à propager la foi. Les traductions congolaises sont des mouvements vers l'autre, vers la langue de l'autre. Les missionnaires montrent explicitement leur intérêt pour les langues indigènes. Nous songeons, par exemple, aux dictionnaires, grammaires et études linguistiques faits par les missionnaires ${ }^{5}$. L'intérêt pour les langues indigènes n'est évidemment pas innocent. Il est fondamentalement guidé par la propagation de la foi. Jules Van Houtte, par exemple, a non seulement traduit le Catéchisme préparatoire au baptême, il a aussi publié un livre de prières et de chants en lonkundu Nsambo la Njembo nde lonkundu.

$\mathrm{Au}$ lieu de traduire le Catéchisme préparatoire au baptême dans différentes langues du Congo, les missionnaires auraient pu se faciliter la vie en enseignant le catéchisme en français. Ils auraient pu faire d'une pierre deux coups : enseigner le catéchisme et apprendre le français aux populations indigènes. Ils auraient imposé non seulement une vision religieuse occidentale, mais aussi une langue occidentale. L'ouverture vers l'autre aurait été moins grande que celle montrée par les missionnaires-traducteurs.

Une question pourrait être posée à propos de la remarque que nous venons de faire. Pourquoi les missionnaires flamands auraientils appris le français aux indigènes ? Pourquoi pas le néerlandais ? Cette situation bizarre pourrait s'expliquer par la situation linguistique à la fin du dix-neuvième et au début du vingtième siècle. À cette époque, le français était la langue dominante en Belgique. Le rapport de force entre le français et le néerlandais a été transposé au Congo.

\footnotetext{
${ }^{5}$ Pour le lonkundo, nous renvoyons aux travaux du missionnaire Gustaaf Hulstaert (Melsele 1900 - Bamanya 1990).

${ }^{6}$ Ibeke: Misio katoliko S. Teresia, 1921.
} 
La position linguistique du néerlandais en Belgique à la fin du dix-neuvième et au début du vingtième siècle pourrait peutêtre expliquer l'ouverture des missionnaires flamands à l'égard des langues congolaises. Le respect de la langue de l'autre est sans doute lié à la position opprimée de la langue maternelle des missionnaires.

Jusqu'à présent, nous n'avons pas encore utilisé le mot 'exil' et nous hésitons à l'utiliser. Le mot est très chargé ; il porte des significations diverses ; il s'emploie entre autres dans des contextes financiers, politiques, psychanalytiques et religieux. Nous nous demandons quelle pourrait être la place du mot 'exil' dans le discours sur la traduction?

Les mots 'Vandredi', 'Samedi', 'Eklesia' et 'Paska', des mots étranges dans la traduction lonkundu que nous avons citée, sont-ils des mots exilés ? Ne faudrait-il pas distinguer exil et étrangeté ? Il serait sans doute abusif de parler ici d'exil. Gonfler l'exil en l'appliquant à tout mot étrange dans une traduction nous paraît problématique. L'exil risque, en fin de compte, de ne plus rien vouloir dire. De plus, l'emploi du mot 'exil' dans un contexte traductologique est sans doute excessif par rapport aux personnes qui sont vraiment en situation d'exil.

Notre contribution pourrait se terminer ici. Nous avons écarté le mot 'exil' du discours sur la traduction. La question est de savoir si cet écartement est justifié. N'est-il pas possible de développer un discours sur les traducteurs exilés ? Les missionnaires-traducteurs n'étaient-ils pas en quelque sorte des exilés, des déracinés, des déplacés ? Ils vivaient à des milliers de kilomètres de leur mère patrie, dans un contexte culturel et linguistique étranger. Un discours sur les traducteurs exilés est intéressant d'un point de vue informatif, mais ce discours est centré sur les sujets traduisants, sur les personnes qui gravitent autour de la traduction. Un discours sur les sujets traduisants évite d'aborder le fond de la chose, l'essence de la traduction.

Le groupe de mots 'essence de la traduction' nous mène aux travaux d'Antoine Berman. L'essence de la traduction est, 
pour Berman, « ouverture, dialogue, métissage, décentrement » (L'épreuve de l'étranger 16). Ces quatre mots pourraient facilement s'appliquer à la traduction lonkundu, dont nous avons cité et commenté un passage. Ces quatre mots sont des images, des métaphores de la traduction. Ils s'ajoutent à la longue liste des métaphores de la traduction. Nous nous demandons si le discours sur la traduction n'est pas sur-métaphorisé. L'emploi de métaphores - exil, par exemple - ne risque-t-il pas de nous éloigner de l'essence de la traduction ? Cette question nous mène à poser une question supplémentaire : un discours non métaphorique sur la traduction, est-il possible?

«[...] l'essence de la traduction est d'être ouverture, dialogue, métissage, décentrement. », est une phrase tirée du livre $L$ 'épreuve de l'étranger d'Antoine Berman. Le titre du livre, $L$ 'épreuve de l'étranger, pourrait lui aussi s'appliquer à la traduction lonkundu. Traduire le Catéchisme préparatoire au baptême en lonkundu est une épreuve, un chemin semé d'obstacles.

Le mot 'épreuve' dans $L$ 'épreuve de l'étranger, ne renvoie pas seulement aux difficultés de la traduction, mais aussi à l'expérience de l'étrange, de l'étranger. Éprouver l'étranger, c'est faire l'expérience de l'étrange, de l'étranger ; c'est traduire.

Pour conclure, nous citons un passage tiré de l'article « La traduction comme épreuve de l'étranger » d'Antoine Berman. Cet article a été publié en 1985 dans la revue Texte, une année après la parution du livre $L$ 'épreuve de l'étranger :

La traduction est "épreuve de l'étranger ». Mais en un double sens. Premièrement, elle instaure un rapport du Propre à l'Étranger, en ce qu'elle vise à nous ouvrir l'œuvre étrangère dans sa pure étrangeté. [...]. Mais en second lieu, la traduction est une épreuve pour l'Étranger lui-même, car elle arrache l'œuvre à son sol-de-langue. Et cette épreuve, souvent pour elle un exil, peut aussi manifester le pouvoir le plus singulier de l'acte de traduire : révéler, de l'œuvre étrangère, son noyau le plus originel, le plus enfoui, le plus 
propre, mais également le plus « lointain». (« La traduction comme épreuve de l'étranger » 67)

Nous vous laissons, sans doute un peu perplexes, avec cette citation, inscrite en filigrane dans cette contribution. Il faudra préciser les termes que Berman utilise : L'Étranger renvoie à la culture source ; le Propre à la culture cible. La traduction est un mouvement de l'Étranger, la culture source, vers le Propre, la culture cible. On pourrait appliquer ces termes au cas que nous avons traité en guise d'exemple : L'Étranger est la culture chrétienne européenne ; le Propre est la culture lonkundu. Le terme le plus intrigant dans la citation est 'sol-de-langue'. Ce terme est un terme-clé dans la définition que Berman nous donne de l'exil. Exiler, c'est arracher l'original à son sol-de-langue. Le Catéchisme préparatoire au baptême est arraché au sol français. Il faut souligner que c'est la traduction, et non le traducteur, qui arrache l'original à son sol-de-langue. Berman définit l'exil de façon désubjectivée.

Nous ajoutons une dernière citation qui permettra de questionner davantage le rapport entre l'exil et la traduction. Elle est tirée de l'« Aufgabe des Übersetzers » de Walter Benjamin:

[Die] reine Sprache, die in fremde gebannt ist, in der eigenen zu erlösen, die im Werk gefangene in der Umdichtung zu befreien, ist die Aufgabe des Übersetzers. (« Die Aufgabe des Übersetzers » XVI)

Le lien entre ce passage et celui tiré de « La traduction comme épreuve de l'étranger » est évident. Berman affirme que la traduction est un exil pour l'œuvre originale. Dans l' « Aufgabe des Übersetzers », le pur langage (reine Sprache) est exilé (gebannt) dans la langue étrangère et il est emprisonné dans l'œuvre originale. La langue étrangère, l'œuvre originale sont les lieux de l'exil ; la propre langue et la traduction sont les lieux de la révélation et de la libération. 
Pour Benjamin toutes les langues (le français, le lonkundu, le néerlandais...) sont liées. Toutes les langues convergent vers un point : le pur langage. Le mouvement vers le pur langage est réalisé par les langues. Il n'est pas réalisé par les sujets utilisant la langue. La conception de Benjamin est une conception fondamentalement désubjectivée.

Les textes originaux (le Catéchisme préparatoire au baptême, par exemple) ont un tout autre rapport au pur langage que les traductions. Les originaux ne s'intéressent pas au pur langage ; les traductions, par contre, sont les témoins privilégiés du mouvement vers le pur langage. Elles indiquent la distance entre la propre langue, la langue cible (le lonkundu, par exemple) et le pur langage. Les originaux n'ont pas ce pouvoir indicateur. Benjamin renverse le rapport hiérarchique traditionnel entre l'original et la traduction. La traduction a un pouvoir que l'original n'a pas. Elle montre, rend visible le mouvement de la langue. Elle a une fonction révélatrice et libératrice.

\section{Références}

S.A. Catéchisme préparatoire au baptême développant les quatre points fondamentaux de la foi traduit en langue Bobangi. Bruxelles: Polleunis et Ceuterick imprimeurs, 1898. [Trad.: Van Ronslé, C.].

S.A. Catéchisme préparatoire au baptême traduit en la langue des Baluba. Bruxelles: Polleunis et Ceuterick imprimeurs, 1898. [Trad.: Garmyn, J.].

S.A. Catéchisme préparatoire au baptême traduit en langue du Bas-Congo. Bruxelles: Polleunis et Ceuterick imprimeurs, 1900. [Trad.: Van Ronslé, C.]. 
S.A. Catéchisme préparatoire au baptême. Nouvelle-Anvers [Makanza]: Impr. Sœurs Francisc. Mission du Sacré-Cœur, 1911.

S.A. Catéchisme préparatoire au baptême traduit dans la langue des Nkundu (du Lac Léopold II). Bruxelles: Imprimerie Fern. De Ryck, 1912.

Benjamin, Walter. «Die Aufgabe des Übersetzers » Charles Baudelaire Tableaux parisiens Deutsche Übertragung mit einem Vorwort über die Aufgabe des Übersetzers. Heidelberg: Verlag von Richard Weissbach (1923): VI-XVII.

Berman, Antoine. L'épreuve de l'étranger. Culture et traduction dans l'Allemagne romantique. Paris: Éditions Gallimard, 1984.

. «La traduction comme épreuve de l'étranger» Texte. Revue de critique et de théorie littéraire 4 (1985): 67-81.

Mpia Bekina, Jacques. L'évangélisation du Mai-Ndombe au Congo-Kinshasa: Histoire, difficultés présentes et inculturation. Paris: L'Harmattan, 2010.

Recebido em: 28/08/2017

Aceito em: 14/10/2017

Publicado em janeiro de 2018

Winibert Segers is affiliated to KU Leuven and to the UCO/Université d'Angers (France). He lectures in French and Dutch on administrative, cultural and medical texts. He completed a doctorate on the untranslatability of writings by the French philosopher, Jacques Derrida. He publishes in the following research areas: translation evaluation, translation didactics, translation ethics, translation philosophy, translation theory and translations of travelogues. Leuven, Flanders, Belgium. E-mail: winibert.segers@kuleuven.be

Henri Bloemen is Associate professor Faculty of Arts of KU Leuven. Translation and Intercultural Transfer, Campus Sint-Andries Antwerp. Leuven, Flanders, Belgium. E-mail: henri.bloemen@kuleuven.be 